\section{(2) OPEN ACCESS}

\begin{abstract}
- Additional material is published online only. To view please visit the journal online (http://dx.doi.org/10.1136/ thoraxjnl-2018-212675).
\end{abstract}

For numbered affiliations see end of article.

\section{Correspondence to} Dr Danielle B Cohen, Infection, Immunity \& Cardiovascular Disease, University of Sheffield, Sheffield S10 2TN, UK: Danielle.Cohen@sheffield.ac.uk

Received 3 October 2018 Revised 13 September 2019 Accepted 20 September 2019 Published Online First 1 November 2019

\title{
Delivery of long-term-injectable agents for TB by lay carers: pragmatic randomised trial
}

\author{
Danielle B Cohen, ${ }^{1,2,3}$ Kuzani Mbendera, ${ }^{4}$ Hendramoorthy Maheswaran, 2,5,6 \\ Mavuto Mukaka, ${ }^{2,7,8}$ Helen Mangochi, ${ }^{2}$ Linna Phiri, ${ }^{2}$ Jason Madan, ${ }^{6}$ Geraint Davies, ${ }^{2,9}$ \\ Elizabeth Corbett, ${ }^{2,10}$ Bertel Squire ${ }^{3}$
}

\begin{abstract}
Background People with recurrent or drug-resistant TB require long courses of intramuscular injections. We evaluate a novel system in which patient-nominated lay carers were trained to deliver intramuscular injections to patients in their own homes.
\end{abstract}

Methods A pragmatic, individually randomised noninferiority trial was conducted at two hospitals in Malawi. Adults starting TB retreatment were recruited. Patients randomised to the intervention received home-based care from patient-nominated lay people trained to deliver intramuscular streptomycin. Patients receiving standard care were admitted to hospital for 2 months of streptomycin. The primary outcome was successful treatment (alive and on treatment) at the end of the intervention.

Results Of 456 patients screened, 204 participants were randomised. The trial was terminated early due to futility. At the end of the intervention, 97/101 (96.0\%) in the hospital arm were still alive and on treatment compared with $96 / 103(93.2 \%)$ in the home-based arm (risk difference $-0.03(95 \% \mathrm{Cl}-0.09$ to 0.03$)$; $p$ value 0.538$)$. There were no differences in the proportion completing 8 months of anti-TB treatment; or the proportion experiencing 2-month sputum culture conversion. The mean cost of hospital-based management was US\$1546.3 per person, compared to US\$729.2 for home-based management. Home-based care reduced risk of catastrophic household costs by $84 \%$.

Conclusions Although this trial failed to meet target recruitment, the available data demonstrate that training patient-nominated lay people has potential to provide a feasible solution to the operational challenges associated with delivering long-term-injectable drugs to people with recurrent or drug-resistant TB in resource-limited settings, and substantially reduce costs. Further data under operational conditions are required.

Trial registration number ISRCTN05815615.

\section{BACKGROUND}

Each year, approximately 700000 people are treated for recurrent TB and a further 480000 for multidrug-resistant TB (MDR-TB). ${ }^{1}$ Treatment regimens for both of these groups currently involve long courses of daily injectable agents. In the case of TB retreatment, patients in countries with low prevalence of MDR-TB and no access to drug susceptibility testing have been prescribed WHO 'Category II retreatment regimen'. ${ }^{2}$ This is an 8 -month course

\section{Key messages}

What is the key question?

- Can patient-nominated lay carers provide intramuscular injections at home to patients requiring long-term-injectable agents for the treatment of TB?

What is the bottom line?

- This model of delivering care to patients receiving daily intramuscular drugs for TB could be feasible and cost saving for both users and providers.

Why read on?

- Training lay carers to give intramuscular injections to patients in their own homes may present a novel opportunity to improve the delivery of care to patients with recurrent or drug-resistant TB.

of oral antituberculous agents with the addition of intramuscular streptomycin for the first 60 days. Most patients with MDR-TB still receive at least 8 months of injectables ${ }^{3}$ and even newer shortened regimens for MDR-TB include 4 months of injectable agents. ${ }^{4}$

Traditionally, patients have been admitted to hospital to receive injections, but long admissions are expensive for both users and providers ${ }^{5-7}$ and associated with acquisition of nosocomial infections. ${ }^{8} 9$ More recently, communitybased approaches to delivering parenteral drugs have been encouraged. Models of delivery have involved either patients travelling daily from the community to a health facility, or a professional health worker visiting patients in their homes. Both models pose significant operational challenges, ${ }^{10}$ and a potential solution is for injections to be given to patients at home by a person living with them or close by.

Over recent years in industrialised countries, programmes of outpatient parenteral antibiotic therapy have been successfully instituted. ${ }^{11-13}$ Evidence suggests that these services are safe, well received by users and highly cost effective. ${ }^{14}$ Patients with diabetes in non-industrialised countries are prescribed insulin and successfully administer subcutaneous injections at home ${ }^{15}$; however, 
no model of carer-administered intramuscular treatment has yet been developed in a resource-limited setting.

In Malawi, 'guardians' have a well-established essential role in caring for people admitted to hospital. They are usually family members or friends who accompany patients, and perform a variety of tasks including basic care, assisting with medications and advocacy. ${ }^{16}$ The aim of this trial was to evaluate a novel method of delivering long-term injectables for $\mathrm{TB}$ in which patient-nominated lay people (guardians) were trained to administer daily intramuscular injections to patients in their own homes.

\section{METHODS}

Trial design and study participants

We conducted a pragmatic, individually randomised trial of hospital versus home-based care during the intensive phase of TB retreatment. Participants randomised to the intervention received home-based care from guardians trained to deliver intramuscular streptomycin. Participants randomised to receive standard care were admitted to hospital for 60 days, as was practice in Malawi.

The trial was conducted at two large hospitals in MalawiQueen Elizabeth Central Hospital (QECH) in Blantyre and Bwaila Hospital in Lilongwe. Consecutive patients registering for TB retreatment were recruited. Individuals were eligible if they were $\geq 16$ years old, able to provide informed consent and able to identify a suitable guardian. People were excluded if they were identified as having MDR-TB or rifampicin-resistant TB; pregnant; or not planning to stay in the area.

\section{Study procedures}

Standard practice in Malawi at the time of the trial was to admit all patients requiring streptomycin for the duration of the course. Patients admitted to hospital to start retreatment were identified each weekday by reviewing the TB and ward registers. Given the pragmatic nature of this trial, the diagnosis of TB was based on clinician assessment in usual operational conditions. Patients were asked to nominate a guardian for training. Consent was obtained separately from both patients and guardians.

A study nurse trained guardians in the technique of intramuscular injection, including injection procedure, sterile technique and disposal of sharps. Once the guardian was able to perform injections safely, they underwent a structured competency assessment (see online supplementary file 1). Participants remained on the ward until randomisation.

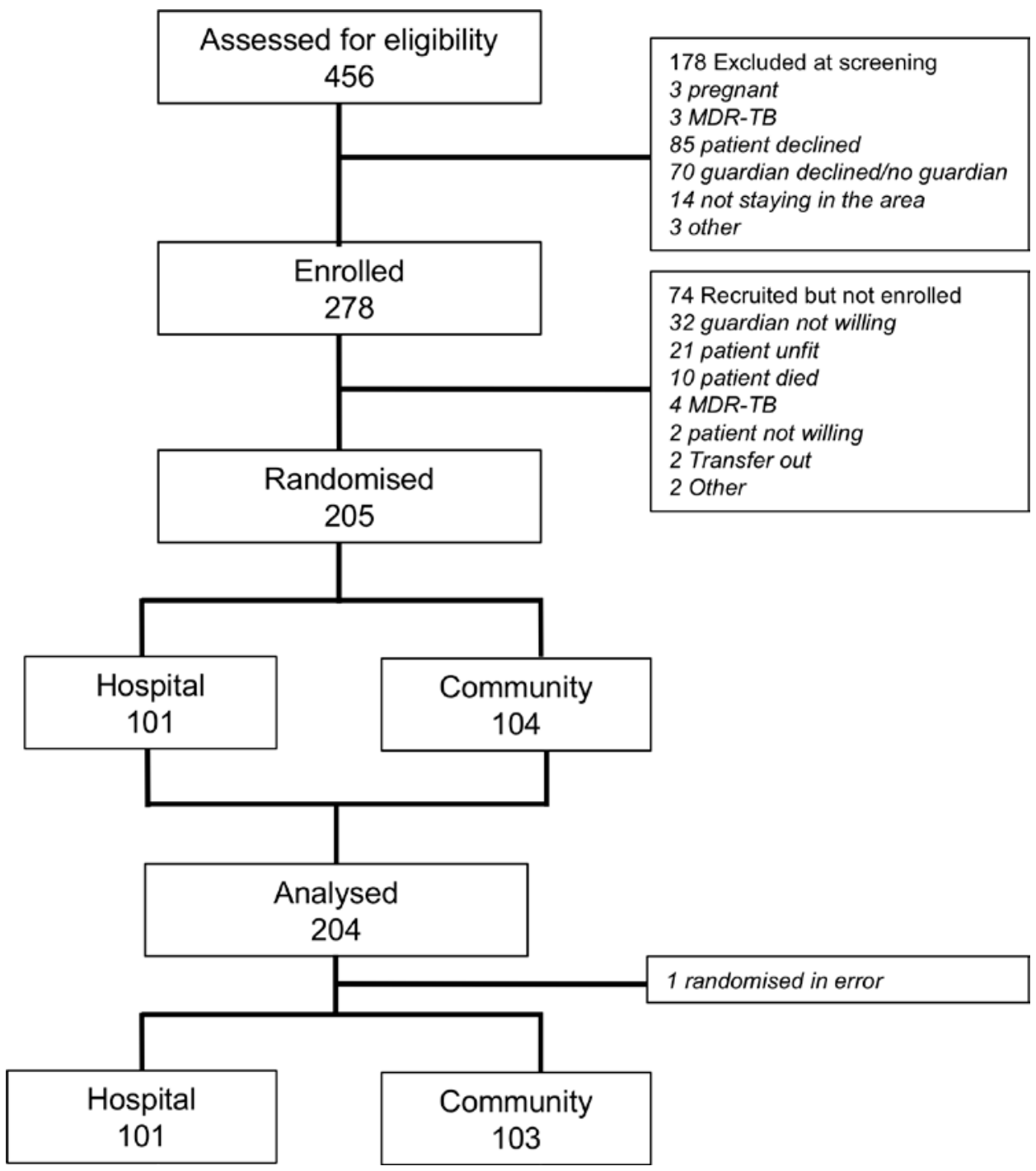

Figure 1 Trial recruitment. MDR-TB, multidrug-resistant TB. 
Table 1 Baseline characteristics of study participants

\begin{tabular}{|c|c|c|c|}
\hline & $\begin{array}{l}\text { Total study population } \\
\mathrm{n}=204(\%)\end{array}$ & $\begin{array}{l}\text { Hospital-based management } \\
\mathrm{n}=101(\%)\end{array}$ & $\begin{array}{l}\text { Home-based management } \\
\mathrm{n}=103(\%)\end{array}$ \\
\hline \multicolumn{4}{|l|}{ Patient characteristics } \\
\hline Patient age (median, IQR) & $36.5(30.0-44.0)$ & $37.0(30.0-44.0)$ & $36.0(30.0-43.0)$ \\
\hline Patient sex (\% male) & $142(69.6)$ & $77(74.8)$ & $65(64.4)$ \\
\hline \multicolumn{4}{|l|}{ TB class } \\
\hline Pulmonary & $182(89.2)$ & $84(83.2)$ & $98(95.2)$ \\
\hline Extrapulmonary & $22(10.78)$ & $17(16.8)$ & $5(4.8)$ \\
\hline \multicolumn{4}{|l|}{ TB category* } \\
\hline Relapse & $99(48.5)$ & $44(43.6)$ & $55(53.4)$ \\
\hline TALTFU & $5(2.5)$ & $2(2.0)$ & $3(2.9)$ \\
\hline Fail & $15(7.4)$ & $5(5.0)$ & $10(9.7)$ \\
\hline Other & $85(41.7)$ & $50(49.5)$ & $35(34.0)$ \\
\hline Sputum culture positive & $80(39.2)$ & $38(37.6)$ & $42(40.8)$ \\
\hline HIV positive & $164(80.4)$ & $87(86.1)$ & $77(74.8)$ \\
\hline Established on ART if HIV positive & $131(80.9)$ & $70(81.4)$ & $61(80.26)$ \\
\hline CD4 count (cells/mm³ median, IQR) & $177(82-423)$ & $161(68-339)$ & $225(117-560)$ \\
\hline \multicolumn{4}{|l|}{ No of previous TB episodes } \\
\hline 1 & $174(85.3)$ & $88(87.1)$ & $86(83.5)$ \\
\hline 2 & $28(13.7)$ & $12(11.9)$ & $16(15.5)$ \\
\hline$>2$ & $2(1.0)$ & $1(1.0)$ & $1(1.0)$ \\
\hline Baseline Karnofsky Score (median, IQR) & $90(80-100)$ & $90(80-90)$ & $90(90-100)$ \\
\hline History of alcohol excess $\dagger$ & $47(23.7)$ & $21(21.1)$ & $26(26.3)$ \\
\hline \multicolumn{4}{|l|}{ Guardian characteristics } \\
\hline Guardian age (median, IQR) & $32(26-40)$ & $33(28-39)$ & $30(25-40)$ \\
\hline Guardian sex (\% male) & $62(30.4)$ & $28(27.7)$ & $34(33.0)$ \\
\hline \multicolumn{4}{|l|}{ Guardian level of education } \\
\hline None & $4(2.0)$ & $4(4.0)$ & $0(0.0)$ \\
\hline Standard 1-4 & $33(16.2)$ & $20(19.8)$ & $13(12.6)$ \\
\hline Standard 5-8 & $52(25.5)$ & $26(25.7)$ & $26(25.2)$ \\
\hline Form 1-2 & $42(20.6)$ & $18(17.8)$ & $24(23.3)$ \\
\hline Form 3-4 & $45(22.1)$ & $20(19.8)$ & $25(24.3)$ \\
\hline University & $28(13.7)$ & $13(12.9)$ & $15(14.6)$ \\
\hline \multicolumn{4}{|l|}{ Guardian relationship to patient } \\
\hline Spouse & $74(36.3)$ & $37(36.6)$ & $37(35.9)$ \\
\hline Sibling & $56(27.5)$ & $28(27.7)$ & $28(27.2)$ \\
\hline Parent & $16(7.8)$ & $9(2.9)$ & $7(6.8)$ \\
\hline Child & $19(9.3)$ & $7(6.9)$ & $12(11.7)$ \\
\hline Aunt/uncle & $14(6.9)$ & $7(6.9)$ & $7(6.8)$ \\
\hline Niece/nephew & $9(4.4)$ & $3(3.0)$ & $6(5.8)$ \\
\hline Friend/neighbour & $16(7.8)$ & $10(9.9)$ & $6(5.8)$ \\
\hline
\end{tabular}

*Standard WHO definitions of TB category: relapse=a patient previously treated for TB, declared cured or treatment completed, who is diagnosed again with smear or culturepositive TB; treatment after failure=a patient who is started on retreatment regimen after having failed previous treatment; treatment after loss to follow-up (TALTFU)=a patient who returns to treatment, positive bacteriologically, following interruption of treatment for 2 or more consecutive months; other previously treated=all previously treated cases that do not fit any of the above definitions http://www.who.int/tb/err/rr_final_forms_en.pdf.

tAs defined by the Alcohol Use Disorders Identification Test. ${ }^{36}$

\section{Randomisation}

Randomisation was performed once the guardian had passed the competency assessment and the patient was fit for discharge. Participants were randomised to receive standard of care (hospital admission) or the intervention (home-based care) during the intensive phase of TB retreatment. Randomisation was carried out by a data team not otherwise involved in the conduct of the trial. Block randomisation in a ratio of $1: 1$ using variable block sizes of 4 or 6 was performed using a computer random number generator to produce an equal allocation ratio. Sequentially numbered opaque sealed envelopes were prepared by an independent person. 
Table 2 TB retreatment outcomes at 2 months

\begin{tabular}{lllll}
\hline & $\begin{array}{l}\text { Hospital-based management } \\
\mathbf{n}=101(\%)\end{array}$ & $\begin{array}{l}\text { Home-based management } \\
\mathbf{n}=103(\%)\end{array}$ & P value & RR (95\% Cl) \\
\hline Alive and on TB treatment & $97(96.0)$ & $96(93.2)$ & 0.538 & $0.97(0.91$ to 1.03$)$ \\
Death & $2(2.0)$ & $7(6.8)$ & 0.170 & $3.43(0.73$ to 16.13$)$ \\
Loss to follow-up & $2(2.0)$ & $0(0.0)$ & 0.244 & Not applicable \\
\hline
\end{tabular}

$\mathrm{RR}$, risk ratio.

\section{Follow-up of study participants}

Participants in both arms were reviewed 1, 3, 5 and 7 weeks post randomisation. Reviews were conducted by a fieldworker with no formal clinical training. At each visit, an assessment for adverse events was performed, including assessments of hearing ${ }^{17}$; urine output; sciatic nerve injury; inspection of the injection site and documentation of new prescribed medications. For participants in the community, all used equipment was collected, and new equipment was delivered. At the first visit, guardians were required to pass another competency test to ensure competency was maintained in the home environment. Adherence was assessed at each visit by self-report and 'vial count' of streptomycin, equivalent to 'pill count' employed to assess adherence to antiretroviral therapy (ART) at HIV clinics in the region. Participants were provided with a form and asked to document each day that streptomycin had been given (see online supplementary file 2), in addition to the 'TB card' provided routinely for monitoring oral treatments. There was no formal assessment of adherence to HIV medications.

\section{Adverse events monitoring}

Given the nature of the intervention, standard definitions were adapted to encompass all possible consequences of home-based care (see online supplementary file 3). All patients presenting with clinical adverse events were reviewed by a study clinician (see online supplementary file 4).

\section{Outcomes}

The primary outcome was successful treatment at the end of the 2-month intervention period. Successful treatment

Table 3 Details of deaths during the intensive phase of TB retreatment

\begin{tabular}{ll}
\hline Details of event & Location \\
\hline Home-based treatment arm & \\
\hline $\begin{array}{l}\text { Stevens Johnsons syndrome } 2 \text { weeks after starting TB treatment, } \\
1 \text { week after starting ART }\end{array}$ & Hospital \\
\hline $\begin{array}{l}\text { End stage heart failure with dilated cardiomyopathy; on treatment } \\
\text { for presumed TB pericarditis }\end{array}$ & Hospital \\
\hline $\begin{array}{l}\text { Acute febrile illness with jaundice; treated for presumed bacterial } \\
\text { sepsis }\end{array}$ & Hospital \\
\hline $\begin{array}{l}\text { Disseminated Kaposi Sarcoma, pancytopenia, ascites, splenomegaly } \\
\text { Headache, dysphasia, hemiparesis; treated for presumed cerebral }\end{array}$ & Hospital \\
toxoplasmosis & \\
\hline $\begin{array}{l}\text { Cryptococcal meningitis } \\
\text { Sudden death }\end{array}$ & Hospital \\
\hline Hospital-based treatment arm & Community \\
\hline $\begin{array}{l}\text { Died while receiving bowel prep for colonoscopy; presumed } \\
\text { electrolyte imbalance }\end{array}$ & Hospital \\
\hline \begin{tabular}{l} 
Jaundice; presumed drug-induced liver injury \\
\hline
\end{tabular}
\end{tabular}

was defined as all those 'still alive and on treatment having completed 2 months streptomycin injections'. Unsuccessful treatment included all patients died, lost to follow-up or outcome unknown. ${ }^{18}$

Predefined secondary endpoints included 2-month sputum culture conversion; programmatic TB outcome after 8 months of treatment; Karnofsky score at 2 months and mental health status at 2 months using a standard Self-Reporting Questionnaire (SRQ) validated in the local language. ${ }^{19}$

\section{Statistical analysis}

The study was powered to detect non-inferiority in the intervention arm. It was assumed that $87 \%$ of patients receiving standard of care and $82 \%$ of patients in the community would be alive and on treatment at the end of the 60-day intervention period, based on a review of records we performed of QECH data in 2012. The sample size was calculated based on a non-inferiority margin of $6 \%$. Using a one-sided alpha at a level of 0.05 , in order to achieve a power of $80 \%$, it was calculated that a sample size of 268 would be required. A single planned interim analysis was conducted after 130 had been recruited.

Primary analysis was based on intention to treat. Secondary per-protocol analysis was planned as a sensitivity analysis, and for this purpose protocol deviation was defined as any participant randomised to receive home-based management who was not discharged by the time they completed 60 days of streptomycin.

Efficacy outcomes are presented as proportions and compared using the Fishers exact test. The risk difference (RD) for unsuccessful treatment outcome was estimated with 95\% CIs. Risk ratios (RRs) with 95\% CIs are presented for key secondary outcomes. There were no planned subgroup analyses. Analysis was performed using STATA V.12.1.

A within-trial cost-consequence analysis ${ }^{20}$ was undertaken from the societal perspective and included provider and user costs (see online supplementary file 5). Catastrohic cost was defined as healthcare costs equivalent to $10 \%$ of annual household income. Written informed consent was obtained from all study participants if literate, or with the signature of an independent witness if illiterate.

\section{RESULTS}

\section{Study participants}

Between June 2013 and February 2015, 456 patients starting TB retreatment regimen were screened. Of those screened, 278 $(60 \%)$ had guardians who began training to administer streptomycin. After further withdrawals during the period of training, a total of 204 participants were randomised (figure 1). One patient was excluded from the analysis as they were randomised in error prior to being declared fit for discharge by a clinician. One hundred and forty-four were enrolled in Blantyre and the remaining 60 in Lilongwe.

A meeting of the Trial Steering Committee was held after 200 patients had been recruited, at which it was decided to stop recruitment of the trial early due to futility. The event rate in the 
Table 4 TB retreatment outcomes at 8 months

\begin{tabular}{|c|c|c|c|c|}
\hline & Hospital-based management $n=79(\%)$ & Home-based management $n=83(\%)$ & $P$ value & $\begin{array}{l}\text { RR } \\
(95 \% \mathrm{Cl})\end{array}$ \\
\hline Successful treatment & $61(77.2)$ & $67(80.7)$ & 0.700 & 1.05 (0.89 to 1.23$)$ \\
\hline Cure & $26(32.9)$ & $41(49.4)$ & 0.039 & 1.50 (1.02 to 2.20$)$ \\
\hline Complete & $35(44.3)$ & $26(31.3)$ & 0.106 & 0.71 (0.47 to 1.06$)$ \\
\hline Fail & $1(1.2)$ & $1(1.2)$ & 1.000 & 0.95 (0.06 to 14.96$)$ \\
\hline Death & $11(13.9)$ & $13(15.7)$ & 0.827 & 1.12 (0.54 to 2.36$)$ \\
\hline Loss to follow-up & $4(5.1)$ & $2(2.4)$ & 0.434 & 0.48 (0.09 to 2.53$)$ \\
\hline Transfer out & $2(2.5)$ & $0(0.0)$ & 0.236 & Not applicable \\
\hline
\end{tabular}

$\mathrm{RR}$, risk ratio.

trial was lower than the 0.13 which had been projected: 0.0645 in the intervention arm and 0.0426 in the control arm, so it was projected that in order to establish non-inferiority, at least 600 participants would be required, and that this was not feasible given the resources available.

Characteristics of the study participants are summarised in table 1. The median age of patients was 36.5 years (IQR 30.044.0 years), and $69.6 \%$ were men. The majority $(89.2 \%)$ had pulmonary $\mathrm{TB}$, classified most commonly as relapse $(48.5 \%)$ or 'other' (41.7\%). The HIV prevalence was $80.4 \%$ and $131 / 164$ (80.9\%) were on ART. The median length of time from start of TB treatment to discharge in the home-based group was 12 days (IQR 8-17 days).

\section{Successful completion of the intensive phase of TB treatment}

At the end of the intensive phase of treatment, 97 (96.0\%) of patients in the hospital arm and 96 (93.2\%) of patients in the home-based arm were still alive and on treatment (RD -0.03 (95\% CI -0.09 to 0.03 ); p value 0.538 ). Only an intention-totreat analysis was performed, as all randomised patients received the form of treatment to which they are assigned. There were seven deaths in the home-based arm, and two in the hospital arm (RR 3.43; 95\% CI 0.73 to 16.13) (table 2). Deaths were reviewed at a meeting of the Data and Safety Monitoring Board, and it was concluded that none were either directly or indirectly related to the intervention. Of the seven deaths which occurred in the home-based arm, six occurred after the patient had been readmitted to hospital (table 3). Two patients were lost to follow-up from hospital-based care, but none were lost to follow-up from home-based care.

\section{Secondary outcomes}

End of TB treatment outcomes

Before the close of the study, 162 patients had registered TB treatment outcomes through the National TB Programme. There was no significant difference in the proportion of patients

Table 5 Adverse events during the intensive phase of TB retreatment

\begin{tabular}{ll}
$\begin{array}{l}\text { Hospital-based } \\
\text { management } \\
\mathrm{n}=101\end{array}$ & $\begin{array}{l}\text { Home-based } \\
\text { management } \\
\mathrm{n}=103\end{array}$ \\
56 & 34 \\
8 & 5 \\
6 & 0 \\
0 & 5 \\
2 & 0 \\
0 & 0 \\
\hline
\end{tabular}

who successfully completed treatment in the home-based arm $(80.7 \%)$ compared with the hospital arm $(77.2 \%)$ of the trial (table 4).

Two-month sputum culture conversion

Of 80 patients who had sputum culture positive for Mycobacterium tuberculosis at the start of treatment, sputum culture was performed in 43 who were able to provide a sample after 2 months of treatment. In the group receiving home-based care, $21 / 23(91.3 \%)$ went from sputum culture positive to sputum culture negative at 2 months, compared with 15/20 (75.0\%) who culture converted in the hospital-based care group (RR 1.22 (95\% CI 0.91 to 1.61 ); p value 0.222 ).

Karnofsky score at the end of the intensive phase of treatment The median Karnofsky score at the end of the intervention period was $100 \%$ in both groups. Of patients still alive at the end of the intensive phase of treatment, $84.4 \%$ in the homebased group and $79.2 \%$ in the hospital group had a Karnofsky score of $100 \%$.

Mental health status at the end of the intensive phase of treatment All but one of the 21 patients identified as having common mental health disorder at baseline no longer screened positive on the SRQ after 2 months of treatment. The patient who continued to screen positive had received hospital-based care.

Table 6 Costs of home-based management in the intensive phase of TB retreatment

\begin{tabular}{|c|c|c|}
\hline & $\begin{array}{l}\text { Hospital-based } \\
\text { management } \\
n=33\end{array}$ & $\begin{array}{l}\text { Home-based } \\
\text { management } \\
\mathrm{n}=32\end{array}$ \\
\hline $\begin{array}{l}\text { Cost indicators } \\
\text { Total cost } 2014 \text { US\$ }\end{array}$ & 47936.6 & 21875.6 \\
\hline $\begin{array}{l}\text { Outcome indicators } \\
\text { Number of patients completing } \\
\text { intensive phase retreatment }\end{array}$ & 31 & 30 \\
\hline $\begin{array}{l}\text { Cost-difference indicators } \\
\text { Cost per patient completing } \\
\text { intensive phase retreatment } 2014 \\
\text { US\$ * }\end{array}$ & $\begin{array}{l}1546.3 \\
(833.8-2764.1)\end{array}$ & $\begin{array}{l}729.2 \\
(341.5-1589.1)\end{array}$ \\
\hline $\begin{array}{l}\text { Incremental cost difference } \\
\text { Cost per patient completing } \\
\text { intensive phase retreatment } 2014 \\
\text { US\$ }\end{array}$ & -817.1 & \\
\hline
\end{tabular}

Cohen DB, et al. Thorax 2020;75:64-71. doi:10.1136/thoraxjnl-2018-212675 
Table 7 Total user costs of hospital-based and home-based management during the intensive phase of TB retreatment

\begin{tabular}{|c|c|c|c|}
\hline & \multicolumn{3}{|l|}{ Mean cost US dollars $(95 \% \mathrm{Cl})$} \\
\hline & Hospital-based management & Home-based management & Mean difference* \\
\hline \multicolumn{4}{|l|}{ Patient costs } \\
\hline \multicolumn{4}{|l|}{ During admission } \\
\hline Direct medicalt & $0.7(0.2$ to 1.2$)$ & $0.4(0.0$ to 0.7$)$ & $0.3(-0.2$ to 1.0$)$ \\
\hline \multicolumn{4}{|l|}{ Direct non-medical } \\
\hline Food & 69.8 (58.4 to 81.2 ) & 26.7 (21.5 to 31.8$)$ & 43.1 (30.5 to 55.7 ) \\
\hline Transport & 3.6 (1.6 to 5.7$)$ & $1.6(0.5$ to 2.6$)$ & $2.1(-0.1$ to 4.3$)$ \\
\hline Linen & $0.7(0.1$ to 1.3$)$ & $1.1(-0.1$ to 2.3$)$ & $-0.5(-1.8$ to 0.9$)$ \\
\hline Other out of pocket & $11.9(7.1$ to 16.7$)$ & 4.7 (3.2 to 6.2$)$ & $7.2(2.1$ to 12.4$)$ \\
\hline Indirect $\ddagger$ & 133.5 (111.6 to 155.4$)$ & $37.0(28.7$ to 45.3$)$ & 96.5 (73.2 to 119.7$)$ \\
\hline \multicolumn{4}{|l|}{ Health facility post discharge } \\
\hline Direct medical and non-medical & - & $1.1(0.3$ to 2.0$)$ & $-1.1(-2.0$ to -0.3$)$ \\
\hline Indirect & - & $0.3(0.1$ to 0.5$)$ & $-0.8(-1.8$ to 0.1$)$ \\
\hline Total patient costs & 220.2 (189.8 to 250.8$)$ & $72.9(60.5$ to 85.3$)$ & $147.4(114.1$ to 180.6$)$ \\
\hline \multicolumn{4}{|l|}{ Guardian costs } \\
\hline \multicolumn{4}{|l|}{ During admission } \\
\hline \multicolumn{4}{|l|}{ Direct non-medical } \\
\hline Food & 17.7 (11.1 to 24.2$)$ & $13.6(9.2$ to 17.9$)$ & $4.1(-3.5$ to 11.7$)$ \\
\hline Transport & $10.0(5.6$ to 14.3$)$ & $6.4(4.1$ to 8.8$)$ & $3.5(-1.2$ to 8.2$)$ \\
\hline Linen & $0.1(-0.1$ to 0.2$)$ & 0.1 (0.0 to 0.3$)$ & $0.0(-0.3$ to 0.2$)$ \\
\hline Other out of pocket & $2.9(1.1$ to 4.7$)$ & $1.6(0.7$ to 2.5$)$ & $1.3(-0.7$ to 3.4$)$ \\
\hline Indirect & $20.6(8.4$ to 32.9$)$ & $5.2(2.8$ to 7.7$)$ & $15.4(3.1$ to 27.7$)$ \\
\hline \multicolumn{4}{|l|}{ Health facility post discharge } \\
\hline Direct non-medical & - & $1.0(-0.6$ to 2.7$)$ & $-1.0(-2.7$ to 0.6$)$ \\
\hline Indirect & - & $0.8(-0.1$ to 1.8$)$ & $-0.8(-1.8$ to 0.1$)$ \\
\hline Total guardian costs & 51.3 (31.8 to 70.8 ) & 28.6 (21.5 to 36.2$)$ & $22.4(2.2$ to 42.7$)$ \\
\hline Total user costs & 271.6 (234.4 to 308.7 ) & 101.8 (85.1 to 118.4 ) & 169.8 (128.9 to 210.7$)$ \\
\hline
\end{tabular}

*Bootstrapped estimates of mean differences and $95 \% \mathrm{Cl}$.

tDrugs and investigations purchased privately if unavailable in the public facility.

‡Lost income.

\section{Adverse events}

There were 56 adverse events in 36 patients receiving hospitalbased care and 34 adverse events in 20 patients receiving home-based care (table 5). One patient reported a missed dose of streptomycin and two patients missed a single dose by 'vial count', but there was no episode which met the predefined criteria for an adherence event. There were no needle stick injuries in either arm. There were eight serious adverse events in the hospital arm and five in the home-based arm. Both adverse events resulting in disability were a consequence of ototoxicity.

\section{Economic evaluation}

Data about user costs were available for 188 trial participants, and data about provider costs were collected for a subgroup of 65 participants (32 receiving home-based care and 33 receiving hospital-based care).

The total cost of hospital-based management was US $\$ 1546.3$ per person compared with US\$729.2 per person who received home-based management, giving a cost difference of US\$ -817.1 (table 6). The total mean cost to users in the hospital arm was US\$271.6 (234.4-308.7), compared with US\$101.8 (85.1-118.4) for those who receiving home-based care (mean difference 169.8 ; 95\% CI 128.9 to 210.7 ) (table 7). Total provider costs were US\$498.0 (425.6-570.4) per patient who received home-based care, compared with US\$1100.3 (1040.81159.8 ) per patient who received hospital-based care (see online supplementary file 6).

The risk of catastrophic household costs was $34.1 \%$ for participants in the home-based arm and $85.9 \%$ for those managed in hospital (RR 0.40 ; 95\% CI 0.29 to 0.54 ) (see online supplementary file 7). Applying a definition of catastrophic cost at $20 \%$ of annual household income, home-based management was associated with an $11.4 \%$ risk of catastrophic cost whereas hospital-based management was associated with a $62.0 \%$ risk of catastrophic cost (RR 0.16 ; 95\% CI 0.08 to 0.41 ) (see online supplementary file 7). The reduction in risk of catastrophic cost was seen irrespective of wealth quartile, gender or HIV status.

\section{DISCUSSION}

This trial failed to meet target recruitment and was therefore insufficiently powered to demonstrate non-inferiority of the intervention; however, the available data do not demonstrate a difference in clinical outcomes between people receiving injectable treatments for TB delivered by lay carers and the traditional hospital-based model of care. Additionally, catastrophic patient costs were largely avoided by home-based care. 
The RD between the two groups in the primary outcome was only $3 \%$. However, the CIs around this estimate reached $9 \%$; therefore, non-inferiority cannot be concluded at the predefined margin of $6 \%$. These figures are open for discussion. First, a non-inferiority margin of $6 \%$ was conservative, with many other trials setting margins of $10 \%-15 \% .21-24$ Second, the point estimate of $3 \%$ difference in risk is low and the absolute risk of patients failing to complete 2 months of home-based retreatment was only $7 \%$, much lower than previous estimates of death or loss to follow-up during the first 2 months of TB retreatment.

There were seven deaths in the home-based arm of the study, compared with only two in the hospital arm. The mortality rate was not statistically different between the two groups; however, the circumstances of these deaths still need to be carefully examined. A higher mortality may have been as a direct consequence of guardians administering injections, but none of the deaths were related to unsafe administration of an intramuscular injection such as sepsis. Additionally, there is no evidence that adherence to treatment in the home-based arm was a problem, as both measures of adherence suggested very few missed doses of streptomycin; the rate of sputum culture conversion at 2 months was similar in both groups; and all of the deaths were due to conditions other than TB. Another possible reason for an increased death rate in the community is that patients at home had less medical attention during the intensive phase of treatment. Some evidence for this may be suggested by the higher number of adverse events in the hospital arm. However, any prescription of medication by a physician was classified as an adverse event and it is well recognised that overprescription of antibiotics and other drugs by clinicians to patients in hospital is common..$^{25} 26$ An indirect effect of home-based care also seems unlikely given that, of the seven deaths in people randomised to the homebased arm, six happened only following readmission to hospital. In 5 out of 6 of these patients, the death took place only after they had been receiving appropriate treatment in hospital for at least 7 days. If this model of care is to be more widely adopted, it will require close ongoing evaluation.

There was no difference between the two study arms in terms of successful 8 -month completion of TB treatment $(80.7 \%$ in the home-based model vs $77.2 \%$ in the hospital-based model), which is operationally the most significant outcome from the perspective of global TB control. Additionally, the rate of 8 -month cure was significantly higher $(49.4 \%$ vs $32.9 \%$, RR 1.50 (1.02 to 2.20; $\mathrm{p}$ value 0.039$)$ ) and the rate of 2 -month culture conversion was non-significantly higher in the home-based arm (91.3\% in the home vs $75.0 \%$ in the hospital). The mechanisms by which home-based care may be contributing to improving some outcome indicators may be through more ownership of health decisions, fewer economic consequences and ultimately better adherence to treatment. ${ }^{27} 28$

The study has a number of limitations. Most importantly, the trial was stopped early because of futility, which impacts the strength of the data. Efforts had been made to predict the expected event rate by conducting a review of the QECH TB register prior to starting the study, but the observed event rate was lower than expected. It is likely that the main reason for this was the requirement for participants to have a guardian able and willing to undertake delivery of the injectable. This resulted in exclusion before randomisation of 102 patients (see figure 1). Patients without guardians or with guardians unwilling to take on the responsibility of injecting may have been less likely to have stable lifestyles. In this way, the trial probably recruited a more stable cohort of patients than those assessed in the record review. Additionally, it is common for outcomes in a trial setting to be better than in routine practice due to increased input from the study team. ${ }^{29} 30$ Although a proportion of patients were not recruited because they were never clinically stable enough to be discharged, it was never intended for the intervention to be applicable to all patients prescribed TB retreatment, and even under routine conditions only those well enough to go home would be considered for home-based management. Finally, the study was conducted at only two sites in Malawi, both in large urban areas, and so results are not necessarily generalisable to other contexts.

The trial also has a number of strengths. As far as possible, the study was pragmatic and provides data for policy-makers that are relevant to routine operational conditions ${ }^{31}{ }^{32}$, for example: reviews conducted by research staff with experience equivalent to that of a health surveillance assistant ${ }^{27}$; eligibility criteria including all patients clinically fit for discharge without additional investigations which would not be available in routine practice; no therapeutic drug monitoring to assess adherence in the community; and a primary outcome measure which is routinely recorded in TB reporting systems. Additionally, a trialbased economic evaluation provides robust data demonstrating that home-based management is associated with significant reductions in both provider and user costs, and qualitative evaluation (published elsewhere ${ }^{33}$ ) demonstrates the acceptability of lay carers delivering injectables.

The reduction in provider costs from US $\$ 1100$ to US $\$ 489$ potentially has an enormous impact on health systems in countries like Malawi, where the total expenditure on health per capita is US\$93. ${ }^{28}$ Additionally, the intervention reduced the risk of catastrophic household costs $\geq 10 \%$ by $60 \%$, and the risk of catastrophic household costs $\geq 20 \%$ by $84 \%$. There are now strong data demonstrating that household catastrophic costs in relation to $\mathrm{TB}$ are associated with poorer clinical TB outcomes, ${ }^{34}$ and one of the targets of the WHO EndTB strategy is that "no TB-affected families should experience catastrophic costs due to TB' ${ }^{35}$ This study was underpowered for clinical outcome, and another large randomised controlled trial would be costly and time consuming. However, the data demonstrate compelling health economic benefits for both providers and users. If $\mathrm{TB}$ programmes consider adopting this model, further data should be collected under operational conditions in order to monitor ongoing clinical safety and economic benefit.

Recent guidelines encourage a move away from the use of long-term-injectable agents for TB. However, the complete phase out of Category II regimen is still dependent on the availability of rapid molecular-based drug-susceptibility testing and resolution of the debate surrounding the best treatment for isoniazid monoresistant TB. Similarly, despite recent communications from WHO, the global use of fully oral regimens for all people with MDR-TB is still some way off given the financial and operational issues which still surround these regimens.

If targets to reduce catastrophic household expenditure associated with TB are to be met, the issue of how to deliver longterm injectable agents to stable patients in the community needs to be addressed. This study presents a novel method of administering injections to patients in their homes as part of their longterm TB care. Training patient-nominated lay people to deliver injectables may offer a sustainable opportunity for home-based management of patients with recurrent or drug-resistant $\mathrm{TB}$ in resource-limited settings but further operational data to support the findings of this study are needed.

\section{Author affiliations}

${ }^{1}$ Infection, Immunity \& Cardiovascular Disease, University of Sheffield, Sheffield, UK ${ }^{2}$ Clinical Department, Malawi Liverpool Wellcome Clinical Research Programme, Blantyre, Malawi 
${ }^{3}$ Department of Clinical Sciences, Liverpool School of Tropical Medicine, Liverpool, UK ${ }^{4}$ Malawi National TB Programme, Lilongwe, Malawi

${ }^{5}$ Institute of Psychology, Health and Society, University of Liverpool, Liverpool, UK

${ }^{6}$ Division of Health Sciences, University of Warwick, Warwick, UK

${ }^{7}$ Mahidol Oxford Tropical Medicine Research Unit, Mahidol University, Bankok, Thailand

${ }^{8}$ Centre for Tropical Medicine and Global Health, University of Oxford, Oxford, United Kingdom

${ }^{9}$ Institute of Infection and Global Health, University of Liverpool, Liverpool, United Kingdom

${ }^{10}$ Department of Clinical Research, LSHTM, London, UK

Acknowledgements The authors would like to thank all patients and guardians who participated so enthusiastically in this trial. They acknowledge the contributions of George Musowa, Jimmy Chavula, Malumbo Ngoma, Melifa Mphasa and Nyembezi Chincombe. They thank the team in the TB laboratory at the Malawi College of Medicine for their hard work, and are grateful for the support received from the dedicated staff of the TB wards at QECH and Bwaila Hospitals.

Contributors DBC designed and implemented the study, analysed the data and wrote the first draft of the manuscript. KM coordinated implementation and data collection at the Lilongwe site. HM and JM advised on the collection and analysis of data for the health economic evaluation. HM provided additional data for the hospital costing. HM and LP were involved in overall study design, implemented the project, managed data collection and provided participant care at the Blantyre and Lilongwe sites, respectively. GD, EC and BS provided supervisory support throughout project design, data collection and analysis. All authors contributed to preparation of the final manuscript.

Funding This work was conducted using funding from a Wellcome Trust grant held by D.B. Cohen.

Disclaimer The Wellcome Trust had no role in the design of the study; collection, analysis and interpretation of data; or writing the manuscript.

Competing interests None declared.

Patient consent for publication Obtained.

Ethics approval The study received ethical approval from the ethics committees of The Liverpool School of Tropical Medicine and The University of Malawi College of Medicine.

Provenance and peer review Not commissioned; externally peer reviewed.

Data availability statement Data are available in a public, open access repository.

Open access This is an open access article distributed in accordance with the Creative Commons Attribution 4.0 Unported (CC BY 4.0) license, which permits others to copy, redistribute, remix, transform and build upon this work for any purpose, provided the original work is properly cited, a link to the licence is given, and indication of whether changes were made. See: https://creativecommons.org/ licenses/by/4.0/.

\section{REFERENCES}

1 Global Tuberculosis Report: World Health Organization, 2016

2 Treatment of tuberculosis guidelines. 4th edn. Geneva, Switzerland: World Health Organization, Dept. ST, 2010.

3 Companion Handbook to the WHO guidelines for the programmatic management of drug-resistant tuberculosis. Geneva: World Health Organization, 2014.

4 WHO treatment guidelines for drug-resistant tuberculosis. World Health Organization, 2016

5 Floyd K, Skeva J, Nyirenda T, et al. Cost and cost-effectiveness of increased community and primary care facility involvement in tuberculosis care in Lilongwe district, Malawi. Int J Tuberc Lung Dis 2003;7:S29-37.

6 Moalosi G, Floyd K, Phatshwane J, et al. Cost-effectiveness of home-based care versus hospital care for chronically ill tuberculosis patients, Francistown, Botswana. Int J Tuberc Lung Dis 2003;7:S80-5.

7 Okello D, Floyd K, Adatu F, et al. Cost and cost-effectiveness of community-based care for tuberculosis patients in rural Uganda. Int J Tuberc Lung Dis 2003;7:S72-9.

8 Allegranzi B, Bagheri Nejad S, Combescure C, et al. Burden of endemic health-careassociated infection in developing countries: systematic review and meta-analysis. Lancet 2011;377:228-41.

9 Nardell E, Dharmadhikari A. Turning off the spigot: reducing drug-resistant tuberculosis transmission in resource-limited settings. Int J Tuberc Lung Dis 2010;14:1233-43.
10 Weiss P, Chen W, Cook VJ, et al. Treatment outcomes from community-based drug resistant tuberculosis treatment programs: a systematic review and meta-analysis. BMC Infect Dis 2014;14:333

11 Török ME, Chapman ALN, Lessing MPA, et al. Outpatient parenteral antimicrobial therapy: recent developments and future prospects. Curr Opin Investig Drugs 2010;11:929-39.

12 Subedi S, Looke DFM, McDougall DA, et al. Supervised self-administration of outpatient parenteral antibiotic therapy: a report from a large tertiary hospital in Australia. Int J Infect Dis 2015;30:161-5.

13 Pajarón M, Fernández-Miera MF, Allende I, et al. Self-administered outpatient parenteral antimicrobial therapy (S-OPAT) for infective endocarditis: a safe and effective model. Eur J Intern Med 2015;26:131-6.

14 Chapman ALN, Dixon S, Andrews D, et al. Clinical efficacy and cost-effectiveness of outpatient parenteral antibiotic therapy (OPAT): a UK perspective. J Antimicrob Chemother 2009:64:1316-24.

15 Cohen DB, Allain TJ, Glover S, et al. A survey of the management, control, and complications of diabetes mellitus in patients attending a diabetes clinic in Blantyre, Malawi, an area of high HIV prevalence. Am J Trop Med Hyg 2010;83:575-81.

16 Hoffman M, Mofolo I, Salima C, et al. Utilization of family members to provide hospital care in Malawi: the role of hospital guardians. Malawi Med J 2012;24:74-8.

17 Torres-Russotto D, Landau WM, Harding GW, et al. Calibrated finger rub auditory screening test (CALFRAST). Neurology 2009;72:1595-600.

18 Definitions and reporting framework for tuberculosis - 2013 revision (updated December 2014). 2014: World Health Organization, 2014

19 Stewart RC, Kauye F, Umar E, et al. Validation of a Chichewa version of the selfreporting questionnaire (SRQ) as a brief screening measure for maternal depressive disorder in Malawi, Africa. J Affect Disord 2009;112:126-34.

20 Drummond MF, Sculpher MJ, Torrance GW, et al. Methods for the economic evaluation of health care programmes. 3rd edn. Oxford University Press, 2005.

21 Molefi M, Chofle AA, Molloy SF, et al. AMBITION-cm: intermittent high dose AmBisome on a high dose fluconazole backbone for cryptococcal meningitis induction therapy in sub-Saharan Africa: study protocol for a randomized controlled trial. Trials 2015; 16:276.

22 Perez-Molina JA, Rubio R, Rivero A, et al. Dual treatment with atazanavir-ritonavir plus lamivudine versus triple treatment with atazanavir-ritonavir plus two nucleos $(\mathrm{t})$ ides in virologically stable patients with HIV-1 (SALT): 48 week results from a randomised, open-label, non-inferiority trial. Lancet Infect Dis 2015;15:775-84.

23 Shrestha R, Krishnan A, Altice FL, et al. A non-inferiority trial of an evidence-based secondary HIV prevention behavioral intervention compared to an adapted, abbreviated version: rationale and intervention description. Contemp Clin Trials 2015;44:95-102

24 Hernandez AV, Pasupuleti V, Deshpande A, et al. Deficient reporting and interpretation of non-inferiority randomized clinical trials in HIV patients: a systematic review. PLOS One 2013:8:e63272.

25 Davey $P$, Brown $E$, Charani $E$, et al. Interventions to improve antibiotic prescribing practices for hospital inpatients. Cochrane Database Syst Rev 2013:CD003543.

26 Charani E, Castro-Sánchez E, Holmes A. The role of behavior change in antimicrobial stewardship. Infect Dis Clin North Am 2014;28:169-75.

27 Kok MC, Namakhoma I, Nyirenda L, et al. Health surveillance assistants as intermediates between the community and health sector in Malawi: exploring how relationships influence performance. BMC Health Serv Res 2016;16:164.

28 Malawi country profile. World Health Organization.

29 van der Wouden JC, Blankenstein $\mathrm{AH}$, Huibers MJH, et al. Survey among 78 studies showed that Lasagna's law holds in Dutch primary care research. J Clin Epidemio 2007:60:819-24

30 Puffer S, Torgerson D, Watson J. Evidence for risk of bias in cluster randomised trials: review of recent trials published in three general medical journals. BMJ 2003;327:785-9

31 Thorpe KE, Zwarenstein M, Oxman $A D$, et al. A pragmatic-explanatory continuum indicator summary (PRECIS): a tool to help trial designers. Can Med Assoc J 2009; 180:E47-57.

32 Treweek S, Zwarenstein M. Making trials matter: pragmatic and explanatory trials and the problem of applicability. Trials 2009;10:37

33 Cohen DB, Phiri $\mathrm{M}$, Banda $\mathrm{H}$, et al. A qualitative evaluation of hospital versus community-based management of patients on injectable treatments for tuberculosis. BMC Public Health 2018;18:1127.

34 Wingfield T, Boccia D, Tovar M, et al. Defining catastrophic costs and comparing their importance for adverse tuberculosis outcome with multi-drug resistance: a prospective cohort study, Peru. PLoS Med 2014;11:e1001675.

35 Global strategy and targets for tuberculosis prevention, care and control after 2015 Geneva, Switzerland: World Health Organisation, 2013.

36 Babor TF, Higgins-Biddle JC, Saunders JB, et al. The alcohol use disorders identification test. Geneva: World Health Organization, 2001 